\title{
Listen and talk to the older patients: a critical emancipatory reflection on the practice of communication issues
}

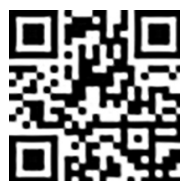

Discussion

Qiao-Miao Zenga ${ }^{a}$ Lian-Xiang $\mathrm{He}^{\mathrm{b}, *}$

aDepartment of Oncological Radiotherapy, XiangYa Hospital, Central South University, Changsha, Hunan 410000, China

${ }^{b}$ Nursing Administration Department, XiangYa Hospital, Central South University, Changsha, Hunan 410000, China

Received: 21 October 2018; Accepted: 22 December 2018; Published: 20 March 2019

Abstract: Objective: Reflection is a process of deliberating thinking and examining one's practice in the past and thereby encouraging nurses to make improvements in future care delivery. This work outlines a piece of reflection involving the practice of communication between nurses and the elderly, to emancipate the authors from their constraints, help to find values as practitioners, and gain a greater understanding of the nurse-patient relationship.

Methods: Using Smyth's four-stage model as a guide, as well as empirical and theoretical knowledge on nurse-patient relationships, this paper presents a deep reflection on the relationship that the authors developed with elderly patients and their families, encountered during the practice as a nurse. By applying the four main stages consisting of describe, inform, confront, and reconstruct, this model enabled the authors to frame, describe the practice issue, and explore the meaning behind it, which helps to facilitate a structured reflection. Results: Critical emancipatory reflection, in association with the Espoused theory and Theory-in-use, as well as reflexivity, critical social theory, and hegemony, was applied to uncover the various power relationships and constraining forces in the authors' practice involved in communicating with the elderly, such as the underlying false consciousness, hegemony, hidden assumptions, influential values, and dominant power structure, which are subtle and persuasive. By applying this process of critical reflection, transformative practice could be achieved. Conclusions: The process of critical reflection facilitated the development of the abilities required to develop and maintain the nursepatient relationship. It helps to enhance the care of old patients and their families, which illuminates the future nursing practice.

Keywords: emancipatory • reflection • older patients $•$ communication $\bullet$ nurse-patient relationship $\bullet$ person-centered care

(c) Shanxi Medical Periodical Press.

\section{Introduction}

Reflective practice is a process for examining practitioners' actions to gain new insights, enabling practitioners to engage in continuous learning. ${ }^{1}$ Nowadays, the ability to continuously and systematically reflect upon practice is regarded to be essential in professional practice. ${ }^{2}$
It enables professionals to develop the awareness of assumptions in order to close the gap between what is espoused and what is enacted in their practice, which is extremely beneficial for nurses to improve in both personal and professional levels. ${ }^{3}$ Critical reflection is a process by which individuals identify and question the assumptions underpinning their action to develop

How to cite this article: Zeng QM, He LX. Listen and talk to the older patients: a critical emancipatory reflection on the practice of communication issues. Front Nurs. 2019; 1: xx-xx. 
alternative behaviors. ${ }^{4}$ As for critical emancipatory reflection, it can lead to transformative action by freeing practitioners from assumptions and oppressive forces that limit them and their practice. ${ }^{5}$

\section{Method}

In this paper, the critical emancipatory reflection, as well as reflexivity, critical social theory, and hegemony, will be applied to uncover various power relationships and constraining forces in the authors' practice involved in communicating with older patients. Smyth's model ${ }^{6}$ will be chosen to assist critical emancipatory reflection. The four-stage process of the model will facilitate the identification of personal beliefs, influences, constraints, and power relationships in this practice issue. It supports the author in developing an awareness of personal power in challenging and changing dominant power structures and helps the author to achieve new perspectives and ways of acting to illuminate future nursing practice. ${ }^{5}$ To begin with, this paper will briefly describe the authors' practice issue and then explore their hidden personal beliefs and the broader theoretical factors in order to assess how these factors affect their practice and constrain their values and intentions. ${ }^{5}$ Finally, reconstruction of this reflection will be discussed regarding the impacts that this process of critical reflection has on the authors own practice, and implications for future practice are proposed.

\section{Results}

\subsection{Describe}

When older patients were admitted to the hospital, I always sought older patients' disease information from their family members. Eventually, these older patients showed their unwillingness to express their needs to me, even though I was there and could be reached for help. Sometimes, they even did not take my instructions. This practice issue was chosen as the focus of my reflection since this situation directly caused hesitation, doubt, or perplexity as my emotional responses. It is of great value to explore the meanings behind this issue to fulfill person-centered care delivery to older patients. Fook and Gardner ${ }^{3}$ describe that the fundamental part of reflection involves making sense of experience in order to inform future practice, which is usually triggered by emotional responses. After eliciting complex emotional responses within me, the assumptions underpinning my taken-for-granted action of preferring to communicate with families can be revealed.

\subsection{Inform}

By applying reflexivity, I was aware of my influence in the way creating knowledge from practice experience, which helps me discover the impacts of my beliefs, assumptions, and behaviors on the issue..$^{3,7}$ In this case, a negative nurse-patient relationship was established between older patients and me, as I failed to make older patients understand my nursing role of being there for them. Besides, their refusal to obey the "rule" of what I suggested for them indicated their suspect attitudes toward my professional authority. At that time, I felt frustrated and confused, since I always make efforts to establish a good relationship with my patients. It seems as if my choice to interact with older patients' families is an action to avoid the possible failure when interacting with patients themselves. Actually, most nurses would like to communicate with families or limit communication to focusing on tasks, rather than directly interacting with older patients. ${ }^{8}$ Possibly, somewhere deep inside me, I hold the belief that older patients have some deficits in their intelligence and memory, that they cannot describe their symptoms as clearly and accurately as their families, and it is difficult for them to understand my words. According to Dionigi, ${ }^{9}$ my assumptions and generalization about how older patients at or older than a certain age should behave, without any consideration of individual differences, is a typical stereotype of aging and ageism. Obviously, this ageism attitude held by me prevented me from interacting and communicating with older patients in my nursing practice.

Meanwhile, I felt embarrassed and upset, since I failed to explore older patients' hidden and unsatisfied needs, which is in contrast with person-centered care delivery, resulting in potential risks in maintaining patient safety. ${ }^{10}$ Usually, health professionals spend less time on older patients' psychosocial issues, since their focus is mainly on older patients' physical illness. In addition, older patients are less likely to raise their concerns, resulting in a lack of awareness of depression in older people. ${ }^{11,12}$ In my practice issue, the psychological and emotional needs of older patients have not been explored and identified when they were admitted to hospital. It seems as if I am willing to deliver personcentered care to older patients, yet various constraints prevent me from achieving this. Person-centered care is considered a standard of care, placing patients at the center of the delivery of care, which needs patients' engagement in making decisions and nurses' provision of both psychological and practical support to them. ${ }^{13}$ However, communication, which is one of the essential means to provide person-centered care, cannot be found in my care delivery for older patients. ${ }^{14}$ Actually, communication provides a chance for health-care 
providers to pay attention to the patient as a whole in the interaction process. ${ }^{15}$ When I only ask information from families, it is hard for me to recognize older patients psychological needs to deliver person-centered care. Nonetheless, the heavy workloads and shortage of nurses in the nursing work environment contributed to the busy and public nature of health-care setting. It can be extremely challenging for professionals to create an environment to initiate good communication with older patients. ${ }^{16}$

\subsection{Confront}

After informing the emotions and subconscious beliefs in my practice issue, it is essential for me to identify the theories related to power that exist in my nursing role, organization, older patients, and families. By purposefully developing assumptions and revealing hidden power through critical reflection, one could exercise the practice differently and achieve transformative change. ${ }^{17}$ Critical social theory was applied to help unearth various constraining forces underpinning the social interaction between older patients and me., ${ }^{3,5}$ The theory of hegemony helped me recognize the ideological meanings through the social struggle for power and dominance in my story, which involves the social hierarchy between older patients and nurses, how nurses struggle amid the policies of the hospital, and the paternalism that exists in Chinese families. ${ }^{5}$

Historically, the communication problems that nurses encounter with older patients arise from societal discrimination and stereotyping. ${ }^{18}$ The stereotype label on older patients is derived from my previous experience, because when I interacted with older patients, they usually ignored my instructions, responded with silence, or were withdrawn, indicating a failure of both trust and the respectful relationship established between us, which can be seen as a deterrent to my authority as a professional. However, when I communicated with older patients' families, the conversation became fluent, effective, and friendly. Explicitly, the values of respect, trust, caring, and responsibility came from my education and professional background. When I worked in some wards that were not so busy, I actively embodied these values in establishing relationships with patients at work, and these values helped me develop a friendly relationship with patients. I believe that as a nurse, I should be responsible to my clients and provide person-centered and quality care to them. ${ }^{19}$ By conducting person-centered care, not only could the patient's satisfaction improve and involvement increase, but also the well-being of patients and a therapeutic environment can be established. ${ }^{20}$ However, when I was in a busy work environment, finishing the tasks required by nurse managers became a top priority, to ask for patients' information from families is considered a way to avoid waste of time and to improve my work efficiency.

By applying the Espoused theory and Theory-in-use in critical reflection, I learned what values and beliefs I uphold in my life, as well as the gap between these values and what enacted values actually were reflected from my action. ${ }^{4}$ It seems like the pursuit of speed is rooted in my routine work, even though I know the best way to acknowledge older patients' needs is to communicate with themselves rather than families, which contributed to the personal constraint in my practice issue. Communicating with older patients can be challenging because of their memory decline and sensory impairment in relation to age. It is also time consuming since older patients often are accompanied by complicated complaints and problems, which need more time to unravel. ${ }^{12}$ Therefore, to communicate with older patients' families is a convenient and fast way to get better understanding of clients and enhance my work efficiency. Actually, families play a critical role in the health-care system, as they help to facilitate communication between the nurses and patients, as well as improve patients' involvement in their own care. They can also clarify older patients' concerns and reinforce any instructions that are provided to patients. ${ }^{12}$ However, it is unwise to neglect patients and totally rely on families' statements. It is essential to make sure that the older patients are fully involved in the nursing and decision-making process to deliver personcentered care. ${ }^{18}$

The social norms of the hierarchy between nurses and older patients contributed to the social constraint in my story. It has been demonstrated that there exists an unbalanced power dynamic in the nurse-patient relationship, with the patient in a vulnerable position and the nurse in a powerful role. ${ }^{21}$ Nurses are in a position of power due to the fact that they have more influence and authority in the health-care system, have specialist knowledge, and have the privilege to get access to information. ${ }^{22}$ Generally, older patients ask fewer questions and are more likely to defer to the professionals' authority. ${ }^{12}$ In my practice issue, on the contrary, of the stereotype nurse-patient power relationship, the way in which older patients ignored my instructions was read by me as a way of challenging my power. Through critical reflection, I realized that in my work, the social hierarchy between nurses and patients has been deeply rooted in my values. Subconsciously, my preference to ask information from families rather than from older patients is a way to protect my dominant power. Usually, stereotyping older patients is caused by a lack of understanding about the physical changes that happen in older adults. ${ }^{18}$ Normal aging is always accompanied by sensory losses, such as hearing and vision changes, 
leading to the deterioration in communicative abilities. ${ }^{18,23}$ Older patients may experience a decreased capacity to master most areas of his or her life, an increased feeling of helplessness, a loss of self-esteem, and even a loss of life purpose and sense of identity. ${ }^{18}$ Additionally, when patients stay in an unfamiliar environment, they might experience anxiety because of the unfamiliar routines, rules, structures, and the deprived personal power or control. ${ }^{24}$ After understanding the nature of aging and patients' emotional changes during hospitalization, I realized that responding with indifference is a common phenomenon in the communication between nurses and older patients. It is not a deliberate action taken by the older patients to challenge my power. Conversely, these indifferent responses of older patients may be a sign of loss of emotional energy in their life as they are vulnerable, which needs further exploration and care.

Applying the critical social theory allows me to realize the internal and external oppression caused by the prevailing ideas of the culture I live in. ${ }^{25}$ Cultural constraints refer to the factors based on the interpretation of shared rituals, symbols, and practices in society, which determine my work patterns when interacting with older patients. ${ }^{5}$ Influenced by Confucian ethics, providing care to older adults is considered as families' obligation and is a highly regarded cultural belief in Chinese culture, which plays an important role in older adults' and families' life. ${ }^{26}$ With regard to "Xiao", which means filial piety in Confucian, older patients' sons have specific obligations toward a family. Children are expected to respect and care for parents and even take on a decision-making role for them when the parents failed to do that. ${ }^{27}$ In addition, the "sick" role is a common behavior among Chinese patients, which means patients usually take a passive role in illness, and family members are expected to look after patients. ${ }^{28}$ Within the context of Chinese culture, the families hold the value that they have the responsibility to look after older patients. Therefore, when they interact and communicate with health-care professionals involved in older patients' disease, it is common that families are more likely to dominate the consultation and decision-making process to show "Xiao", indicating that families use power over older patients. In my story, the dominant role of families is taken for granted by me. This paternalistic attitude among older patients' families, which is rooted in Chinese families, contributed to my neglect toward older patients' feedback and emotional response.

From the political perspective, providing person-centered care is an essential part to ensure quality care in the health-care system, which is emphasized by both the Chinese Ministry of Health and the Nursing and Midwifery Board of Ireland.29 However, from the economical perspective, the situation of nurse shortage and overloaded work environment caused by the budget shortage in the health-care system makes it extremely difficult for nurses to deliver person-centered care. ${ }^{24}$ Even though I realized the need to provide person-centered care to older patients, it seems as if I act according to the belief that I am powerless to change my action in an overloaded work environment. It is hard for me to spend more time to communicate with older patients when I am in a busy environment, since the work performance is mainly determined by the quantity of routine tasks in my ward, and it is my responsibility to complete all the work in my duty. ${ }^{5}$ As a consequence, the communication with older patients to explore their further needs was ignored in my practice. However, the overloaded work environment and the unstaffed situation in an organization can impede the delivery of care and patient safety. ${ }^{24,30}$ To avoid this, a work environment with sufficient staff is needed to help me explore older patients' needs, with adequate time to communicate with them; moreover, some efficient techniques should be applied to facilitate the communication process so as to make it run smoothly.

\subsection{Reconstruct}

By revealing these ideological influences and hidden assumptions in my practice, I enter the final process of emancipatory reflection, transformation, or reconstructing as described in Smyth' model. ${ }^{17}$ In this practice issue, I was aware that the psychological and emotional needs of older patients should be given great importance and be deeply explored in order to deliver person-centered care. ${ }^{31}$ I should gather more information when I sense that there is a loss of emotional energy, as well as desolation, in older patients. ${ }^{18}$ Besides, I realize that I need to get rid of ageism and develop my interpersonal communicating skills to promote person-centered communication for older patients. After I realized the important role of communication in discovering older patients' emotional and psychological needs, it has become essential for me to pay more attention to these needs by communicating and interacting with older patients themselves rather than relying on their families. Moreover, with the revealing of Chinese families' dominant role in managing older patients' disease trajectory, it is necessary to recognize that both older patients and family members are important parts in the health-care team. Ensuring adequate communication with both of them is especially important for the older patients because of their deficiencies in terms of visual, auditory, and mental acuity. ${ }^{32}$ In the future, whenever families take a dominant role in providing information about older patients' disease to me, I will use my interpersonal skills to involve the older patients in the encounter, even if families interject by answering questions. ${ }^{12}$ 
Besides my personal development, getting support from managers and colleagues in an organization is also of great importance. It is essential for the organization to provide support to nurses, as well as provide them with the chance to get access to resources and opportunities to create an empowering work environment. ${ }^{33}$ Besides, it is also important to employ sufficient staff nurses to liberate them from the shackles of routines. Only in this way can I be free from the daily nursing work, increase the time spent on communicating and interacting with older patients, and then deliver person-centered care in a more flexible, creative, and individualized style. ${ }^{34}$ Furthermore, a professional guidebook on caring for older patients published by the Nursing and Midwifery Board of Ireland in $2015,{ }^{31}$ which illustrates the importance and detailed framework of providing person-centered holistic care to older patients, can also be used to guide my future work with older patients.

\section{Conclusions}

Through critical emancipatory reflective practice, a deep critical reflection on the forces and taken-for-granted assumptions that influence my practice was undertaken. Ultimately, the complex power relationships among

\section{References}

1. Donovan MO. Implementing reflection: insights from pre-registration mental health students. Nurse Educ Today. 2007;27:610-616.

2. Fook J. Reflective practice and critical reflection. In: Lishman J. ed. Handbook for Practice Learning in Social Work and Social Care. 3rd ed. London: Jessica Kingsley Publishers. 2015:363-375.

3. Fook J, Gardner F. Practicing Critical Reflection: A Resource Handbook. Berkshire: Open University Press. 2007.

4. Savaya R, Gardner F. Critical reflection to identify gaps between espoused theory and theory-in-use. Soc Work. 2012;57:145-154.

5. Taylor B. Reflective Practice for Healthcare Professionals. 3rd ed. New York: Open University Press. 2010.

6. Smyth J. Developing and sustaining critical reflection in teacher education. Curr Issues Educ. 1989;9:2-9.

7. Parker Z. Breaking the pattern: from reactive to reflective practice. Int $J$ Ther Rehabil. 2015;22:58-59.

8. Williams CL. Therapeutic Interaction in Nursing. 2th ed. London: Jones \& Bartlett Learning. 2008.

9. Dionigi RA. Stereotypes of aging: their effects on the health of older adults. J Geriatr. 2015:1-9. older patients, families, organization, and me were unearthed, giving me a new sense of informed consciousness to bring about positive personal, political, and social change. ${ }^{5} \mathrm{I}$ am becoming aware that through the development of new insights and knowledge, I possess the personal power to change future practice and the broader environment. Critical reflection is a continual and ongoing process of identifying the problems or issues causing dissonance, in order to change or challenge the situation. ${ }^{35}$ In the future, it is of great importance to make changes through a continual process of critical reflection in my work and daily life. Keeping a reflective journal would be a useful way to maintain ongoing reflection in the future, because of its benefits in terms of increasing self-awareness of experiences via the process of clarifying and reflecting on perceptions, thoughts, and feelings. ${ }^{7}$ Adopting reflective writing will facilitate my development of critical thinking, promote integrated learning, encourage my personal development, and establish a good habit of maintaining ongoing reflection. ${ }^{36}$

\section{Conflicts of interest}

All contributing authors declare no conflicts of interest.
10. Henriksen K, Battles JB, Keyes MA, Grady ML. Advances in Patient Safety: New Directions and Alternative Approaches. Rockville: Agency for Healthcare Research and Quality. 2008. https://www.ahrq.gov/ downloads/pub/advances2/vol3/Advances-TitleCitation_Vol3.pdf. Accessed April 26, 2018.

11. Galloway J. Dignity, values, attitudes, and personcentred care. In: Hindle A, Coates A, eds. Nursing Care of Older People. Oxford: Oxford University Press. 2011:9-22.

12. Hingle ST, Robinson S. Enhancing communication with older patients in the outpatient setting. Semin Med Pract. 2009;12:1-7.

13. Richmond Group of Charities. From Vision to Action Making Patient-Centred Care A Reality. England: The King's Fund. 2012. https://www.kingsfund.org. uk/publications/articles/vision-action-making-patientcentred-care-reality. Accessed February 22, 2018.

14. Jayadevappa R. Patient-centered outcomes research and patient-centered care for older adults: a perspective. Gerontol Geriatr Med. 2017;3:1-4.

15. Constand MK, MacDermid JC, Dal Bello-Haas V, Law M. Scoping review of patient-centered care approaches in healthcare. BMC Health Serv Res. 2014;14:271. 
16. Farrington N, Townsend K. Enhancing nursepatient communication: a critical reflection. $\mathrm{Br} \mathrm{J}$ Nurs. 2014;23:771-775.

17. Gardner F. Working with Human Service Organisations: Creating Connections for Practice. Melbourne: Oxford University Press. 2006.

18. Arnold EC, Boggs KU. Interpersonal Relationships: Professional Communication Skills for Nurses. 7th ed. Missouri: Elsevier Health Sciences. 2015.

19. Health Service Executive. Guiding Framework for the Implementation of Nursing and Midwifery Quality Care-Metrics in the Health Service Executive Ireland. Ireland: Health Service Excusive. 2015.

20. McCance T, McCormack B, Dewing J. An exploration of person-centredness in practice. Online $J$ Issues Nurs. 2011;16:1.

21. Carter MA. Trust, power, and vulnerability: a discourse on helping in nursing. Nurs Clin North Am. 2009;44:393-405.

22. Nursing Council of New Zealand. Guidelines: Professional Boundaries. Nursing Council of New Zealand. 2012. https://www.google.co.uk/\#q=Nursing+ Council+of+New+Zealand,+2012.+Guidelines:+Pro fessional+Boundaries. Accessed April 15, 2018.

23. Lawrence $M$, Kinn $S$. Defining and measuring patient-centred care: an example from a mixed methods systematic review of the stroke literature. Health Expectations. 2012;15:295-326.

24. Zolnierek $C$. The importance of knowing the patient. Issues Ment Health Nurs. 2011;32:392-393.

25. Gardner F. Being Critically Reflective: Engaging in Holistic Practice. New York: Palgrave Macmillan. 2014.

26. Lai DW. Filial piety, caregiving appraisal, and caregiving burden. Res Aging. 2010;32:200-223.

27. Stokes SC, Pan C. Health and Health Care of Chinese American Older Adults. Stanford CA: eCampus Geriatrics. 2010. http://geriatrics.stanford.edu/ wp-content/uploads/downloads/ethnomed/chinese/ downloads/chinese_american.pdf. Accessed April 15, 2018.

28. Dixon B. Cultural Traditions and Healthcare Beliefs of Some Older Adults. Diversity and Immigrant Student Support. Red River College. 2009.

29. Nursing and Midwifery Board of Ireland (NMBI). The Code of Professional Conduct and Ethics for Registered Nurses and Registered Midwives. Nursing and Midwifery Board of Ireland. 2014. https:// www.nmbi.ie/nmbi/media/NMBI/Publications/Codeof-professional-Conduct-and-Ethics.pdf?ext=.pdf. Accessed April 15, 2018.

30. Carayon P, Gurses AP. Nursing workload and patient safety - a human factors engineering perspective. In: Hughes R, ed. Patient Safety and Quality: An Evidence-based Handbook for Nurses. Vol 3. Rockville, MD: Agency for Healthcare Research and Quality. 2008.

31. Nursing and Midwifery Board of Ireland (NMBI). Working with Older People: Nursing Guidance. Nursing and Midwifery Board of Ireland. 2015. https://www.nmbi.ie/nmbi/media/NMBI/Publications/working-with-older-people.pdf?ext=.pdf. Accessed April 15, 2018.

32. Fineberg HV. Retooling for an aging America. Medscape J Med. 2008;10:188.

33. Regan LC, Rodriguez L. Nurse empowerment from a middle-management perspective: nurse managers' and assistant nurse managers' workplace empowerment views. Permanente J. 2011;15:e101-e107.

34. Kelly J. Barriers to achieving patient-centered care in Ireland. Dimensions Crit Care Nurs. 2007;26:29-34.

35. Canada-Phillips S. A physical educators use of Freirean praxis for critical reflection. Phys Educ. 2014;71:635-643.

36. Mariko S. Student teachers' reflective journals on teaching practice experiences. Contemp PNG Stud. 2011;14:67. 